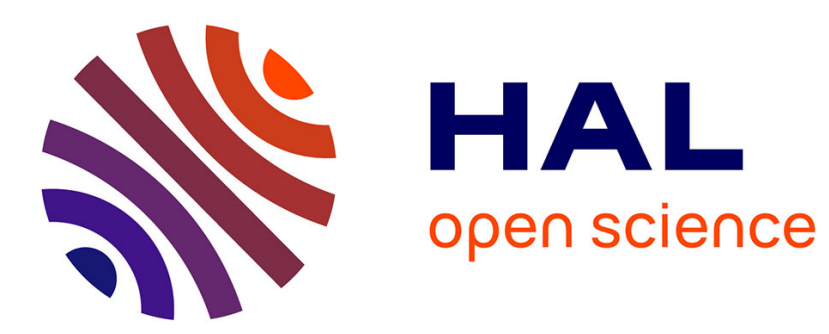

\title{
Two Guaranteed Equilibrated Error Estimators for Harmonic Formulations in Eddy Current Problems
}

Emmanuel Creusé, Yvonnick Le Menach, Serge Nicaise, Francis Piriou, Roberta Tittarelli

\section{- To cite this version:}

Emmanuel Creusé, Yvonnick Le Menach, Serge Nicaise, Francis Piriou, Roberta Tittarelli. Two Guaranteed Equilibrated Error Estimators for Harmonic Formulations in Eddy Current Problems. Computers \& Mathematics with Applications, 2019, 77 (6), pp.1549-1562. 10.1016/j.camwa.2018.08.046 . hal-01955692

\section{HAL Id: hal-01955692 \\ https://hal.science/hal-01955692}

Submitted on 14 Dec 2018

HAL is a multi-disciplinary open access archive for the deposit and dissemination of scientific research documents, whether they are published or not. The documents may come from teaching and research institutions in France or abroad, or from public or private research centers.
L'archive ouverte pluridisciplinaire HAL, est destinée au dépôt et à la diffusion de documents scientifiques de niveau recherche, publiés ou non, émanant des établissements d'enseignement et de recherche français ou étrangers, des laboratoires publics ou privés. 


\title{
Two Guaranteed Equilibrated Error Estimators for Harmonic Formulations in Eddy Current Problems
}

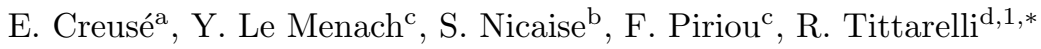 \\ ${ }^{a}$ Laboratoire Paul Painlevé UMR 8524 and EPI RAPSODI INRIA Lille Nord Europe. \\ Université de Lille, Cité Scientifique, 59655 Villeneuve d'Ascq Cedex, France. \\ ${ }^{b}$ Univ. Valenciennes, EA 4015 - LAMAV - FR CNRS 2956, F-59313 Valenciennes, France. \\ ${ }^{c}$ L2EP, Centrale Lille, Arts et Métiers Paris Tech, University Lille, F-59000 Lille, France. \\ ${ }^{d}$ ENSMM, 26 chemin de l'Epithape, 25000 Besançon, and FEMTO-ST Institute, University \\ of Bourgogne Franche-Comté, France.
}

\begin{abstract}
In this paper, two guaranteed equilibrated error estimators are proposed and compared for the 3D harmonic magnetodynamic problem of Maxwell's system. This system is recasted in the classical $\mathbf{A}-\varphi$ potential formulation or, equivalently, in the $\mathbf{T}-\Omega$ potential formulation, and it is solved by the Finite Element method. The first equilibrated estimator presented is built starting from these two complementary problems, the other one is built starting from the $\mathbf{A}-\varphi$ numerical solution uniquely by a flux reconstruction technique. The equivalence between errors and estimators is established. Afterwards, an analytical benchmark test illustrates the obtained theoretical results and a physical benchmark test shows the efficiency of these two estimators.
\end{abstract}

Keywords: A posteriori estimator, eddy current problem, Finite Element Method, Nédélec and Raviart-Thomas elements, time-harmonic analysis, 3D problem

\footnotetext{
${ }^{*}$ Corresponding author

URL: emmanuel.creuse@univ-lille.fr (E. Creusé), yvonnick.le-menach@univ-lille.fr (Y. Le Menach), serge.nicaise@univ-valenciennes.fr (S. Nicaise), francis.piriou@univ-lille1.fr (F. Piriou), roberta.tittarelli@ens $2 m . f r(R$. Tittarelli)

${ }^{1}$ Work realized during an A.T.E.R. contract at Laboratoire Paul Painlevé UMR 8524, Université Lille 1, Cité Scientifique, 59655 Villeneuve d'Ascq Cedex, France.
} 


\section{Introduction}

In this paper we deal with the estimate of the energy error for 3D electromagnetic simulations. In electromagnetism the Finite Element Method is classically used to compute the magnetic and the electric fields. The complexity of the 5 structures, in particular in industrial 3D applications, leads to problems with a huge number of degrees of freedom, which implies long computational times. Thus, in order to get a good compromise between precision and computational times, adapted refinement mesh techniques are performed. There exist different kinds of a posteriori error estimators which indicate the local error, so that they can drive the mesh adaptivity process. For eddy current problems the residual error estimator is often used 1, 2, 3, 4, but the gap between the error and the estimator in unknown, even if they have the same behavior. On the other hand, the equilibrated technique allows us to estimate the distance between the error and the estimator without unknown constants. In this paper we present and compare two equilibrated error estimators for eddy current problems modeled by the so called $\mathbf{A}-\varphi$ formulation. The idea consists in evaluating the gap of the numerical solution with an admissible solution through the discrete nonverification of the constitutive laws. In a magnetostatic framework for example, an admissible field is a magnetic field $\mathbf{H}$ which satisfies Maxwell's equations, but not the constitutive law. Thus, the challenge is to build an admissible field to compare with the discrete solution. This field can be built with the equilibrated approach: one way consists in solving complementary formulations as in [5, 6, 7] for a magnetodynamic framework, another way consists in constructing a field locally starting from the numerical solution [8, 9, 10. Since the global resolution of the complementary problem leads to a computational cost equivalent to the resolution of the original problem, local reconstruction techniques are more and more explorated.

The first estimator presented below is based on the "dual problem technique" which involves the dual formulation $\mathbf{T}-\Omega$. It is therefore available to estimate the sum of the errors of these two possible numerical resolutions, $\mathbf{A}-\varphi$ and 
$\mathbf{T}-\Omega$, see [11] for the complete theoretical analysis. The second one is based on a "flux reconstruction technique" which involves uniquely the $\mathbf{A}-\varphi$ solution, so that it estimates the numerical error of the $\mathbf{A}-\varphi$ resolution only. It is based on reconstructed fluxes for the eddy current allowing us to estimate the electric

35 error. Once these fluxes are available, a magnetostatic numerical resolution provides a magnetic admissible field allowing to estimate the magnetic error. The novelty of the paper is to present the theoretical analysis of this latter estimator, to give some technical details to implement it efficiently and to compare it with the dual estimator above mentioned. Indeed, we adapt and extend the works 40 of [12] (for Laplace equation) and of [13] and [14] (for the electric formulation involving the original electromagnetic fields) to electromagnetic potential formulations.

The paper is organized as follows. Section 2 is devoted to the presentation of the classical $\mathbf{A}-\varphi$ and $\mathbf{T}-\Omega$ formulations and their finite element discretizations as well as to the definitions of energetic errors to estimate. In Section 3 we define the two equilibrated error estimators and state the main results about the equivalence between errors and estimators. Section 4 deals with the detailed proof of the upper bound of the $\mathbf{A}-\varphi$ error by the estimator built from the reconstructed flux technique. Section 5 gives some practical implementation remarks and proposes two numeric tests. In particular, an analytic benchmark test validates the theoretical predictions and a physical numerical test shows the efficiency of these two estimators and allows us to compare them. Section 6 concludes the paper providing some remarks and perspectives.

\section{Analytical and numerical formulations}

Let us consider a bounded simply connected polyhedral domain $D \subset \mathbb{R}^{3}$ with a Lipschitz connected boundary $\Gamma=\partial D . D$ is composed of three subdomains: the source domain $D_{s}$ where the divergence free current density $\mathbf{J}_{s}$ is imposed, the conducting domain $D_{c}$ and non-conducting domain $D_{n c}$. Let us remark 
that $D_{c}$ is supposed bounded and simply connected with a Lipschitz connected boundary $\Gamma_{c}=\partial D_{c}$. The eddy current problem is given by:

$$
\left\{\begin{aligned}
\operatorname{curl} \mathbf{E} & =-j \omega \mathbf{B}, \\
\operatorname{curl} \mathbf{H} & =\mathbf{J}_{s}-\mathbf{J}_{e}, \\
\operatorname{div} \mathbf{B} & =0
\end{aligned}\right.
$$

where $\mathbf{E}$ denotes the electric field, B the magnetic flux, $\mathbf{H}$ the magnetic field, $\mathbf{J}_{s}$ the source term and $\mathbf{J}_{e}$ the eddy current, $j^{2}=-1$ is the unit imaginary number and $\omega$ the pulsation, with the constitutive laws

$$
\mathbf{B}=\mu \mathbf{H} \text { in } D \text { and } \mathbf{J}_{e}=\sigma \mathbf{E} \text { in } D_{c},
$$

where $\mu$ denotes the magnetic permeability and $\sigma$ the electric conductivity. The boundary conditions on $\Gamma$ and $\Gamma_{c}$ are respectively

$$
\mathbf{B} \cdot \mathbf{n}=0 \text { on } \Gamma,
$$

and

$$
\mathbf{J}_{e} \cdot \mathbf{n}=0 \text { on } \Gamma_{c},
$$

where $\mathbf{n}$ stands for the unit outward normal to $D$ or $D_{c}$ depending on the context.

The problem of interest is modeled by the well known $\mathbf{A}-\varphi$ and $\mathbf{T}-\Omega$ formulations, which are reported in the next two sections: the continuous formulations firstly and the numerical approximations secondly. Let us introduce some notations used throughout the paper. On a given domain $\mathcal{D}$, the $L^{2}(\mathcal{D})$ norm is denoted by $\|\cdot\|_{\mathcal{D}}$, and the corresponding $L^{2}(\mathcal{D})$-inner product by $(\cdot, \cdot)_{\mathcal{D}}$. In the case of $\mathcal{D}=D$, the index $D$ is dropped. $H_{0}^{1}(\mathcal{D})$ is the subspace of $H^{1}(\mathcal{D})$ with vanishing trace on $\partial \mathcal{D}$ and

$$
H_{0}(\operatorname{curl}, \mathcal{D})=\left\{\mathbf{F} \in L^{2}(\mathcal{D})^{3}: \operatorname{curl} \mathbf{F} \in L^{2}(\mathcal{D})^{3}, \mathbf{F} \times \mathbf{n}=0 \text { on } \partial \mathcal{D}\right\} .
$$

Finally, in order to ensure later the uniqueness of the fields, let us introduce the 
gauge spaces:

$$
\begin{aligned}
\widetilde{X}(\mathcal{D}) & =\left\{\mathbf{F} \in H_{0}(\operatorname{curl}, \mathcal{D}):(\mathbf{F}, \nabla \xi)_{\mathcal{D}}=0, \forall \xi \in H_{0}^{1}(\mathcal{D})\right\} \\
\widetilde{H^{1}}(\mathcal{D}) & =\left\{f \in H^{1}(\mathcal{D}):(f, 1)_{\mathcal{D}}=0\right\}
\end{aligned}
$$

\subsection{Continuous formulations}

The harmonic $\mathbf{A}-\varphi$ formulation is based on the introduction of a vector potential $\mathbf{A}$ in $D$ and a scalar potential $\varphi$ in $D_{c}$ such that:

$$
\mathbf{B}=\operatorname{curl} \mathbf{A} \text { in } D \text { and } \mathbf{E}=-j \omega \mathbf{A}-\nabla \varphi \text { in } D_{c} .
$$

From system (1), the harmonic $\mathbf{A}-\varphi$ formulation reads:

$$
\begin{aligned}
\operatorname{curl}\left(\mu^{-1} \operatorname{curl} \mathbf{A}\right)+\sigma(j \omega \mathbf{A}+\nabla \varphi) & =\mathbf{J}_{s} \text { in } D, \\
\operatorname{div}(\sigma(j \omega \mathbf{A}+\nabla \varphi)) & =0 \text { in } D_{c},
\end{aligned}
$$

with the boundary conditions, derived from (2)-(3), given by

$$
\mathbf{A} \times \mathbf{n}=0 \text { on } \Gamma \text { and } \sigma(j \omega \mathbf{A}+\nabla \varphi) \cdot \mathbf{n}=0 \text { on } \Gamma_{c} .
$$

The Coulomb gauge on $\mathbf{A}$, namely $\operatorname{div} \mathbf{A}=0$, and the zero mean of the potential $\varphi$ in $D_{c}$ ensure the uniqueness of these potentials. Since $\varphi$ does not make sense in $D_{n c}$, we fix an arbitrary extension of $\varphi$ in the whole domain $D$. This choice does not impact the problem since $\sigma \equiv 0$ in $D_{n c}$. The corresponding weak formulation is given by:

Find $(\mathbf{A}, \varphi) \in \widetilde{X}(D) \times \widetilde{H^{1}}\left(D_{c}\right)$ such that

$$
\begin{aligned}
& \left(\mu^{-1} \operatorname{curl} \mathbf{A}, \operatorname{curl} \mathbf{A}^{\prime}\right)_{D}+j \omega^{-1}\left(\sigma(j \omega \mathbf{A}+\nabla \varphi),\left(j \omega \mathbf{A}^{\prime}+\nabla \varphi^{\prime}\right)\right)_{D_{c}} \\
& =\left(\mathbf{J}_{s}, \mathbf{A}^{\prime}\right)_{D}, \quad \forall\left(\mathbf{A}^{\prime}, \varphi^{\prime}\right) \in \widetilde{X}(D) \times \widetilde{H^{1}}\left(D_{c}\right) .
\end{aligned}
$$

Theorem 2.1 of [2] ensures the existence and uniqueness of the weak solution

${ }_{60}(\mathbf{A}, \varphi)$ of this problem.

Similarly, the harmonic $\mathbf{T}-\Omega$ formulation is based on the introduction of a magnetic source $\mathbf{H}_{s}$ in $D_{s}$, a vector potential $\mathbf{T}$ in $D_{c}$, and a scalar potential $\Omega$ 
in $D$ such that:

$$
\begin{aligned}
& \operatorname{curl} \mathbf{H}_{s}=\mathbf{J}_{s} \text { in } D_{s}, \\
& \operatorname{curl} \mathbf{T}=\mathbf{J}_{e} \text { in } D_{c}, \\
& \mathbf{H}= \begin{cases}\mathbf{H}_{s}-\nabla \Omega & \text { in } D_{n c}, \\
\mathbf{H}_{s}+\mathbf{T}-\nabla \Omega & \text { in } D_{c} .\end{cases}
\end{aligned}
$$

Thus the harmonic $\mathbf{T}-\Omega$ formulation reads:

$$
\begin{aligned}
\operatorname{curl}\left(\sigma^{-1} \operatorname{curl} \mathbf{T}\right)+j \omega \mu(\mathbf{T}-\nabla \Omega) & =-j \omega \mu \mathbf{H}_{s} \text { in } D_{c}, \\
\operatorname{div}(\mu(\mathbf{T}-\nabla \Omega)) & =-\operatorname{div}\left(\mu \mathbf{H}_{s}\right) \text { in } D,
\end{aligned}
$$

where we have fixed an extension of $\mathbf{T}$ in the non conductor domain $D_{n c}$, like what we did for $\varphi$. From (2)-(3), the boundary conditions are given by:

$$
\mathbf{T} \times \mathbf{n}=0 \text { on } \Gamma_{c} \text { and } \mu\left(\nabla \Omega-\mathbf{H}_{s}\right) \cdot \mathbf{n}=0 \text { on } \Gamma .
$$

The uniqueness of the potential is ensured by the Coulomb gauge on $\mathbf{T}(\operatorname{div} \mathbf{T}=$ 0 ) and the zero mean value in $D$ for the potential $\Omega$. The corresponding weak formulation is given by:

Find $(\mathbf{T}, \Omega) \in \widetilde{X}\left(D_{c}\right) \times \widetilde{H^{1}}(D)$ such that

$$
\begin{aligned}
& \left(\sigma^{-1} \operatorname{curl} \mathbf{T}, \operatorname{curl} \mathbf{T}^{\prime}\right)_{D_{c}}+\left(j \omega \mu(\mathbf{T}-\nabla \Omega), \mathbf{T}^{\prime}-\nabla \Omega^{\prime}\right)_{D} \\
& =\left(j \omega \mu \mathbf{H}_{s}, \mathbf{T}^{\prime}-\nabla \Omega^{\prime}\right)_{D}, \forall\left(\mathbf{T}^{\prime}, \Omega^{\prime}\right) \in \widetilde{X}\left(D_{c}\right) \times \widetilde{H^{1}}(D) .
\end{aligned}
$$

Theorem 2.2 from 3 ] ensures the existence and uniqueness of the weak solution $(\mathbf{T}, \Omega)$ of this problem.

\subsection{Numerical formulations}

Let $\mathcal{T}_{h}$ be a regular and conforming mesh made of simplicies, e.g. tetrahedra and $\mathcal{N}_{h}$ the set of the nodes of the mesh. Each element $T$ of $\mathcal{T}_{h}$ belongs either to $D_{c}$ or to $D_{n c}$ and the faces are denoted by $F, h_{T}$ stands for the diameter of the element $T$ and $h=\max _{T \in \mathcal{T}_{h}} h_{T}$ for the mesh size, $\mathbf{n}_{T}$ denotes the unit normal vector to the boundary of $T$ pointing out of $T$ and, for each $F$, we fix $\mathbf{n}_{F}$ as a unit normal vector to $F$. Moreover, $\sigma$ and $\mu$ are supposed to be constant on each tetrahedron. In the following, for a fixed $T \in \mathcal{T}_{h}, \mathbb{P}_{l}(T)$, with $l \in\{0,1\}$, denotes the space of polynomials of degree at most $l$ in $T$ and $\mathcal{D}$ can be $D$ or 
$D_{c}$, depending on the choice of the formulation. Then the approximation spaces are the space of first order edge elements, given by

$$
X_{h}(\mathcal{D})=\left\{\mathbf{F}_{h} \in H_{0}(\operatorname{curl}, \mathcal{D}): \mathbf{F}_{h \mid T} \in \mathcal{N} \mathcal{D}_{1}(T), \forall T \in \mathcal{T}_{h}\right\}
$$

with local Nédélec space

$$
\mathcal{N D}_{1}(T)=\left(\mathbb{P}_{0}(T)\right)^{3}+\left(\mathbb{P}_{0}(T)\right)^{3} \times \mathbf{x},
$$

and the space of first order nodal elements, given by

$$
\Theta_{h}(\mathcal{D})=\left\{\xi_{h} \in H^{1}(\mathcal{D}) ; \xi_{h \mid T} \in \mathbb{P}_{1}(T), \forall T \in \mathcal{T}_{h}\right\} .
$$

The vector fields $\mathbf{A}$ and $\mathbf{T}$ are approximated by first order edge elements and the scalar fields $\varphi$ and $\Omega$ by first order nodal elements. In order to ensure the uniqueness of these fields, we include gauge conditions in the above finite element spaces, so that we define:

$$
\begin{aligned}
& \widetilde{X}_{h}(\mathcal{D})=\left\{\mathbf{F}_{h} \in X_{h}(\mathcal{D}):\left(\mathbf{F}_{h}, \nabla \xi_{h}\right)_{\mathcal{D}}=0, \forall \xi_{h} \in \Theta_{h}^{0}(\mathcal{D})\right\}, \\
& \widetilde{\Theta}_{h}(\mathcal{D})=\left\{f_{h} \in \Theta_{h}(\mathcal{D}):(f, 1)_{\mathcal{D}}=0\right\},
\end{aligned}
$$

where $\Theta_{h}^{0}(\mathcal{D})$ represents the set of functions belonging to $\Theta_{h}(\mathcal{D})$ with vanishing trace on $\partial \mathcal{D}$. The discrete $\mathbf{A}-\varphi$ formulation reads:

Find $\left(\mathbf{A}_{h}, \varphi_{h}\right) \in \widetilde{X_{h}}(D) \times \widetilde{\Theta}_{h}\left(D_{c}\right)$ such that

$$
\begin{aligned}
& \left(\mu^{-1} \operatorname{curl} \mathbf{A}_{h}, \operatorname{curl} \mathbf{A}_{h}^{\prime}\right)_{D}+j \omega^{-1}\left(\sigma\left(j \omega \mathbf{A}_{h}+\nabla \varphi_{h}\right),\left(j \omega \mathbf{A}_{h}^{\prime}+\nabla \varphi_{h}^{\prime}\right)\right)_{D_{c}} \\
& =\left(\mathbf{J}_{s}, \mathbf{A}_{h}^{\prime}\right)_{D}, \quad \forall\left(\mathbf{A}_{h}^{\prime}, \varphi_{h}^{\prime}\right) \in \widetilde{X_{h}}(D) \times \widetilde{\Theta}_{h}\left(D_{c}\right) .
\end{aligned}
$$

Theorem 2.2 of [2] ensures the existence of a unique solution $\left(\mathbf{A}_{h}, \varphi_{h}\right)$. On the other hand, the discrete $\mathbf{T}-\Omega$ formulation reads:

Find $\left(\mathbf{T}_{h}, \Omega_{h}\right) \in \widetilde{X_{h}}\left(D_{c}\right) \times \widetilde{\Theta}_{h}(D)$ such that

$$
\begin{aligned}
& \left(\sigma^{-1} \operatorname{curl} \mathbf{T}_{h}, \operatorname{curl} \mathbf{T}_{h}^{\prime}\right)_{D_{c}}+\left(j \omega \mu\left(\mathbf{T}_{h}-\nabla \Omega_{h}\right), \mathbf{T}_{h}^{\prime}-\nabla \Omega_{h}^{\prime}\right)_{D} \\
& =\left(j \omega \mu \mathbf{H}_{s}, \mathbf{T}_{h}^{\prime}-\nabla \Omega_{h}^{\prime}\right)_{D}, \quad \forall\left(\mathbf{T}_{h}^{\prime}, \Omega_{h}^{\prime}\right) \in \widetilde{X_{h}}\left(D_{c}\right) \times \widetilde{\Theta}_{h}(D) .
\end{aligned}
$$

Theorem 2.4 of [3] ensures the existence of a unique solution $\left(\mathbf{T}_{h}, \Omega_{h}\right)$. 
The goal is to estimate the gap between the continuous and discrete solutions. Indeed, we are interested in the energy norm of the $\mathbf{A}-\varphi$ error $\epsilon_{A, \varphi}$, given by:

$$
\epsilon_{A, \varphi}=\left(\left\|\mu^{-1 / 2} \operatorname{curl} \epsilon_{A}\right\|^{2}+\left\|\omega^{-1 / 2} \sigma^{1 / 2}\left(j \omega \epsilon_{A}+\nabla \epsilon_{\varphi}\right)\right\|_{D_{c}}^{2}\right)^{1 / 2}
$$

where

$$
\epsilon_{A}=\mathbf{A}-\mathbf{A}_{h} \text { and } \epsilon_{\varphi}=\varphi-\varphi_{h}
$$

and, in the energy norm of the $\mathbf{T}-\Omega$ error $\epsilon_{T, \Omega}$, given by:

$$
\epsilon_{T, \Omega}=\left(\left\|\mu^{1 / 2}\left(\epsilon_{T}-\nabla \epsilon_{\Omega}\right)\right\|^{2}+\left\|(\omega \sigma)^{-1 / 2} \operatorname{curl} \epsilon_{T}\right\|_{D_{c}}^{2}\right)^{1 / 2}
$$

where

$$
\epsilon_{T}=\mathbf{T}-\mathbf{T}_{h} \text { and } \epsilon_{\Omega}=\Omega-\Omega_{h}
$$

Let us point out the link between the energy quantities and the original fields. From the FE resolution of the $\mathbf{A}-\varphi$ system, we can define:

$$
\mathbf{B}_{h}=\operatorname{curl} \mathbf{A}_{h} \text { and } \mathbf{E}_{h}=-\left(j \omega \mathbf{A}_{h}+\nabla \varphi_{h}\right),
$$

and from the FE resolution of the $\mathbf{T}-\Omega$ system, we can define:

$$
\mathbf{H}_{h}=\mathbf{H}_{s}+\mathbf{T}_{h}-\nabla \Omega_{h} \text { and } \mathbf{J}_{e, h}=\operatorname{curl}_{h}
$$

Consequently, the $\mathbf{A}-\varphi$ and $\mathbf{T}-\Omega$ errors can be reformulated as:

$$
\epsilon_{A, \varphi}=\left(\left\|\mu^{-1 / 2}\left(\mathbf{B}-\mathbf{B}_{h}\right)\right\|^{2}+\left\|\omega^{-1 / 2} \sigma^{1 / 2}\left(\mathbf{E}-\mathbf{E}_{h}\right)\right\|_{D_{c}}^{2}\right)^{1 / 2}
$$

and

$$
\epsilon_{T, \Omega}=\left(\left\|\mu^{1 / 2}\left(\mathbf{H}-\mathbf{H}_{h}\right)\right\|^{2}+\left\|(\omega \sigma)^{-1 / 2}\left(\mathbf{J}_{e}-\mathbf{J}_{e, h}\right)\right\|_{D_{c}}^{2}\right)^{1 / 2} .
$$

They are both constituted of a sum of the errors on the magnetic energy and ohmic losses. 


\section{A posteriori equilibrated error estimators}

The two mathematical properties defining an optimal error estimator are

70 12:

- The reliability: the estimator $\eta$, computed in the whole domain, gives an upper bound for the error $\epsilon$, computed in the whole domain, of the type $\epsilon \leq$ $C \eta$ up to some higher order terms, where $C$ is a constant independent of the mesh size. This guarantees the control of the error from the estimator.

\subsection{Dual construction method}

Since the $\mathbf{A}-\varphi$ and $\mathbf{T}-\Omega$ formulations are dual formulations, their link can be used to estimate the energy norm error, as already done in the magnetostatic case [15]. Indeed, from the $\mathbf{A}-\varphi$ formulation, a pair of admissible fields is available: the magnetic flux density $\mathbf{B}_{h}$ and the electric field $\mathbf{E}_{h}$. In the same way, the $\mathbf{T}-\Omega$ formulation gives two admissible fields: the eddy current $\mathbf{J}_{e, h}$ and the magnetic field $\mathbf{H}_{h}$. These fields do not satisfy the discrete constitutive laws, so for each mesh element $T$ it is possible to define a local error estimator, denoted by $\eta_{\text {dual,T }}$, evaluating the gap in the $L^{2}$-norm between these fields, as follows:

$$
\eta_{\text {magn }, T}=\left\|\mu^{1 / 2}\left(\mathbf{H}_{h}-\mu^{-1} \mathbf{B}_{h}\right)\right\|_{T}, \eta_{\text {elec }, T}=\left\|(\omega \sigma)^{-1 / 2}\left(\mathbf{J}_{e, h}-\sigma \mathbf{E}_{h}\right)\right\|_{T}
$$

where $\eta_{\text {elec }, T}$ is defined only if $T \subset D_{c}$, and

$$
\eta_{\text {dual }, T}=\left(\sum_{T \in \mathcal{T}_{h}}\left(\eta_{\text {magn }, T}^{2}+\eta_{\text {elec }, T}^{2}\right)\right)^{1 / 2} .
$$


The a posteriori error estimator is globally defined as:

$$
\eta_{\text {dual }}=\left(\sum_{T \in \mathcal{T}_{h}} \eta_{\text {dual }, T}^{2}\right)^{1 / 2} .
$$

The reliability and local efficiency of this estimator are proved in [11, we recall the exact statements in the following:

Theorem 3.1. Let $D$ and $D_{c}$ be simply connected and assume that $\Gamma_{c}$ is connected. Then

$$
\eta_{\text {dual }}^{2}=\epsilon_{A, \varphi}^{2}+\epsilon_{T, \Omega}^{2}+\text { higher order terms. }
$$

Moreover, the following local lower bound for the error holds:

$$
\eta_{\text {dual }, T}^{2} \leq 2\left(\epsilon_{A, \varphi, T}^{2}+\epsilon_{T, \Omega, T}^{2}\right)+\text { higher order terms }
$$

where $\epsilon_{A, \varphi, T}$ and $\epsilon_{T, \Omega, T}$ are the local errors defined locally in the same spirit of tively.

The higher order terms, not present in the magnetostatic case, are the main difference and the hurdle with respect to the static case.

\subsection{Flux reconstruction method}

Another way to build a guaranteed estimator which does not involve a dual formulation consists in starting from one of the dual solutions, let us choose the pair $\left(\mathbf{B}_{h}, \mathbf{E}_{h}\right)$ from the $\mathbf{A}-\varphi$ resolution, and construct an admissible pair of fields $\left(\mathbf{H}_{h}, \mathbf{J}_{e, h}\right)$, which are computed in the most local/efficient way possible. Consequently, the estimated error will be uniquely $\epsilon_{A, \varphi}$. We denote the latter admissible fields with the same notation of the fields involved in the dual construction method since they have the same role of the complementary fields of the $\mathbf{T}-\Omega$ formulation. In the following, let the Raviart-Thomas space of order $l \in\{0,1\}$ in $T$ be

$$
\mathcal{R} \mathcal{T}_{l}(T)=\left(\mathbb{P}_{l}(T)\right)^{3}+\mathbb{P}_{l}(T) \mathbf{x}
$$


and the broken Raviart-Thomas space in $D$ be

$$
\mathcal{R} \mathcal{T}_{l, h}=\left\{\mathbf{F}_{h} \in H(\operatorname{div}, D): \mathbf{F}_{h \mid T} \in \mathcal{R} \mathcal{T}_{0}(T) \forall T \in \mathcal{T}_{h}\right\}
$$

Let us build the admissible fields in two steps.

(i) Since the numerical current density $\sigma \mathbf{E}_{h}$ is not a divergence free field, the idea is to develop an admissible numerical current density $\mathbf{J}_{e, h}$ such that $\operatorname{div} \mathbf{J}_{e, h}=0$. The following construction is inspired from [13, 16]. Let $l_{F} \in \mathbb{P}_{1}(F)$ be a flux such that $l_{F}=0$ if $F \subset \Gamma_{c}$ and for any $T \in \mathcal{T}_{h}: T \subset D_{c}$ such that

$$
\int_{T}-\sigma \mathbf{E}_{h} \cdot \nabla w_{h}=\sum_{F \in \partial T} \int_{F} l_{F}\left(\mathbf{n}_{T} \cdot \mathbf{n}_{F}\right) w_{h}, \quad \forall w_{h} \in \mathbb{P}_{1}(T)
$$

We remark that, evaluating the weak formulation 11 with $\mathbf{A}_{h}^{\prime}=0$ and $\varphi_{h}^{\prime}=$ $\lambda_{\mathbf{x}}$, where $\lambda_{\mathbf{x}}$ represents the $\mathbb{P}_{1}$-conform basis function associated with the node $\mathbf{x} \in \mathcal{N}_{h}$, we obtain

$$
\int_{\omega_{\mathbf{x}}} \sigma \mathbf{E}_{h} \cdot \nabla \lambda_{\mathbf{x}}=0 \quad \forall \mathbf{x} \in \mathcal{N}_{h},
$$

where $\omega_{\mathbf{x}}$ is the set of mesh elements sharing the node $\mathbf{x}$. From this relation the existence of $l_{F} \in \mathbb{P}_{1}(F)$ is ensured, for the full details see Section 6.4 of [12]. Now, $\mathbf{J}_{e, h} \in H\left(\operatorname{div}, D_{c}\right)$ is constructed such that $\mathbf{J}_{e, h} \mid T \in \mathcal{R} \mathcal{T}_{1}(T)$, indeed for each $T \in \mathcal{T}_{h}: T \subset D_{c}$ it is the unique solution of the system

$$
\left\{\begin{array}{l}
\int_{F} \mathbf{J}_{e, h} \cdot \mathbf{n}_{F} q=\int_{F} l_{F} q \quad \forall q \in \mathbb{P}_{1}(F), \quad \forall F \subset \partial T \\
\int_{T} \mathbf{J}_{e, h}=\int_{T} \sigma \mathbf{E}_{h} .
\end{array}\right.
$$

90 For any $T \in \mathcal{T}_{h}: T \subset D_{n c}$ we take the extension $\mathbf{J}_{e, h}=0$ such that $\mathbf{J}_{e, h} \in$ $H(\operatorname{div}, D)$, this is possible having $\mathbf{J}_{e, h} \cdot \mathbf{n}=0$ on $\Gamma_{c}$ as a consequence of 20a and that $l_{F}=0$ for all $F \subset \Gamma_{c}$. Thanks to the continuity of the normal component of $\mathbf{J}_{e, h}, \mathbf{J}_{e, h}$ belongs to $\mathcal{R} \mathcal{T}_{1, h}$.

(ii) From the previous construction we dispose of the divergence free eddy cur-

${ }_{95}$ rent $\mathbf{J}_{e, h}$, then it remains to build the magnetic admissible field $\mathbf{H}_{h}$. Its existence is proved in Theorem 13 of [14], which can be formulated as follows. 
Lemma 3.2. There exists $\mathbf{H}_{h} \in X_{h}$ such that

$$
\operatorname{curl} \mathbf{H}_{h}=\Pi_{h} \mathbf{J}_{s}+\Pi_{h} \mathbf{J}_{e, h},
$$

where $\Pi_{h}$ is a suited projection onto $\mathcal{R} \mathcal{T}_{0, h}$.

For an explicit construction of $\mathbf{H}_{h}$ we can use a classical resolution by the FEM of 21.

In conclusion, this equilibrated estimator, called from now on $\eta_{\text {flux }}$, has the same structure of $\eta_{\text {dual }}$, see (17) and (18), with the difference on the computation of the pair $\left(\mathbf{J}_{e, h}, \mathbf{H}_{h}\right)$. Indeed, globally it is defined as:

$$
\eta_{\text {flux }}=\left(\sum_{T \in \mathcal{T}_{h}} \eta_{\text {flux }, T}^{2}\right)^{1 / 2}=\left(\sum_{T \in \mathcal{T}_{h}}\left(\eta_{\text {magn }, T}^{2}+\eta_{\text {elec }, T}^{2}\right)\right)^{1 / 2},
$$

\section{Guaranteed upper bound for $\eta_{\text {flux }}$}

Lemma 4.1. If $\mathbf{J}_{e, h} \in \mathcal{R} \mathcal{T}_{1, h}$ satisfies 20 in $D_{c}$ and is zero in $D_{n c}$, then $\operatorname{div} \mathbf{J}_{e, h}=0$.

Proof. Since $\operatorname{div} \mathcal{R} \mathcal{T}_{1, h}=\mathbb{P}_{1}\left(\mathcal{T}_{h}\right)=\left\{f \in L^{2}(D): f_{\mid T} \in \mathbb{P}_{1}(\mathcal{T}) \forall T \in \mathcal{T}_{h}\right\}$ (see [17]), we have to prove that $\int_{D} \operatorname{div} \mathbf{J}_{e, h} w_{h}=0$ for any $w_{h} \in \mathbb{P}_{1}\left(\mathcal{T}_{h}\right)$. Let us fix

$$
\begin{aligned}
\int_{D} \operatorname{div} \mathbf{J}_{e, h} w_{h} & =\sum_{T \subset \mathcal{T}_{h}: T \subset D_{c}}\left(-\int_{T} \mathbf{J}_{e, h} \cdot \nabla w_{h}+\int_{\partial T} \mathbf{J}_{e, h} \cdot \mathbf{n}_{T} w_{h}\right) \\
& =-\sum_{T \subset \mathcal{T}_{h}: T \subset D_{c}} \int_{T} \sigma \mathbf{E}_{h} \cdot \nabla w_{h}+\sum_{F \subset \partial T} \int_{F}\left(\mathbf{J}_{e, h} \cdot \mathbf{n}_{F}\right) \mathbf{n}_{T} \cdot \mathbf{n}_{F} w_{h} \\
& =-\sum_{T \subset \mathcal{T}_{h}: T \subset D_{c}} \int_{T} \sigma \mathbf{E}_{h} \cdot \nabla w_{h}+\sum_{F \subset \partial T} \int_{F} l_{F}\left(\mathbf{n}_{T} \cdot \mathbf{n}_{F}\right) w_{h}=0
\end{aligned}
$$


where we have used for the first line element-wise Green's formula, for the second line the properties (20) and the fact that $\mathbf{n}_{F}$ is unitary, and for the third line relation $(19)$. The conclusion follows since this identity is valid for all $w_{h} \in \mathbb{P}_{1}\left(\mathcal{T}_{h}\right)$.

Theorem 4.2. Let us suppose that $\mathbf{J}_{s} \in\left(L^{2}(D)\right)^{3}$ and that $\mathbf{J}_{e, h} \in \mathcal{R} \mathcal{T}_{1, h}$ satisfies (20) and is zero in $D_{n c}$. Then there exists a constant $C>0$ which does not depend on the mesh size (but on the regularity of the mesh) and there exists $\delta \in(0,1]$, which depends on the geometry of $D$ but not on the mesh size $h$, such that the following upper bound holds:

$$
\epsilon_{A, \varphi} \leq \eta_{\text {flux }}+r
$$

where $r$ represents an oscillation term defined by:

$$
r=C \mu_{\max }^{1 / 2}\left(\operatorname{osc}\left(\mathbf{J}_{s}\right)+\operatorname{osc}\left(\mathbf{J}_{e, h}\right)\right)
$$

with $\operatorname{osc}\left(\mathbf{J}_{s}\right)=h^{\delta}\left\|\mathbf{J}_{s}-\Pi_{h} \mathbf{J}_{s}\right\|$ and $\operatorname{osc}\left(\mathbf{J}_{e, h}\right)=h^{\delta}\left\|\mathbf{J}_{e, h}-\Pi_{h} \mathbf{J}_{e, h}\right\|$. For $a$ smooth source term $\mathbf{J}_{s} \in\left(H^{1}(D)\right)^{3}, r$ is consequently a higher order term.

Proof. From definition 12 and remarking that

$$
\left\|\left(\frac{\sigma}{\omega}\right)^{1 / 2}\left(j \omega \epsilon_{A}+\nabla \epsilon_{\varphi}\right)\right\|_{D_{c}}=\left\|\left(\frac{j \sigma}{\omega}\right)^{1 / 2}\left(j \omega \epsilon_{A}+\nabla \epsilon_{\varphi}\right)\right\|_{D_{c}},
$$

we have

$$
\begin{aligned}
\epsilon_{A, \varphi}^{2}= & \int_{D} \mu^{-1} \operatorname{curl}\left(\mathbf{A}-\mathbf{A}_{h}\right) \cdot \operatorname{curl} \overline{\epsilon_{A}}+\int_{D_{c}} \frac{j \sigma}{\omega}\left(j \omega\left(\mathbf{A}-\mathbf{A}_{h}\right)+\nabla\left(\varphi-\varphi_{h}\right)\right) \cdot \overline{\left(j \omega \epsilon_{A}+\nabla \epsilon_{\varphi}\right)} \\
= & \int_{D} \mu^{-1} \operatorname{curl} \mathbf{A} \cdot \operatorname{curl} \overline{\epsilon_{A}}+\int_{D_{c}} \frac{j \sigma}{\omega}(j \omega \mathbf{A}+\nabla \varphi) \cdot \overline{\left(j \omega \epsilon_{A}+\nabla \epsilon_{\varphi}\right)}+\int_{D}\left(\mathbf{H}_{h}-\mu^{-1} \mathbf{B}_{h}\right) \cdot \operatorname{curl} \overline{\epsilon_{A}} \\
& +\frac{j}{\omega} \int_{D_{c}}\left(\sigma \mathbf{E}_{h}-\mathbf{J}_{e, h}\right) \cdot \overline{\left(j \omega \epsilon_{A}+\nabla \epsilon_{\varphi}\right)}+\frac{j}{\omega} \int_{D_{c}} \mathbf{J}_{e, h} \cdot \overline{\left(j \omega \epsilon_{A}+\nabla \epsilon_{\varphi}\right)}-\int_{\Omega} \mathbf{H}_{h} \cdot \operatorname{curl} \overline{\epsilon_{A}} \\
= & \int_{D} \mathbf{J}_{s} \cdot \overline{\epsilon_{A}}-\int_{D} \operatorname{curl} \mathbf{H}_{h} \cdot \overline{\epsilon_{A}}+\frac{j}{\omega} \int_{D} \mathbf{J}_{e, h} \cdot \overline{\left(j \omega \epsilon_{A}+\nabla \epsilon_{\varphi}\right)} \\
& +\frac{j}{\omega} \int_{D_{c}}\left(\sigma \mathbf{E}_{h}-\mathbf{J}_{e, h}\right) \cdot \overline{\left(j \omega \epsilon_{A}+\nabla \epsilon_{\varphi}\right)}+\int_{D}\left(\mathbf{H}_{h}-\mu^{-1} \mathbf{B}_{h}\right) \cdot \operatorname{curl} \overline{\epsilon_{A}}
\end{aligned}
$$

where to pass to the second line we have used definitions of $\mathbf{E}_{h}$ and $\mathbf{B}_{h}$ through potentials $\mathbf{A}_{h}$ and $\varphi_{h}$, see [13, and we have added the quantities $\pm \int_{D} \mathbf{H}_{h}$. 
$\operatorname{curl} \bar{\epsilon}_{A} \pm \frac{j}{\omega} \int_{D} \mathbf{J}_{e, h} \cdot \overline{\left(j \omega \epsilon_{A}+\nabla \epsilon_{\varphi}\right)}$, to pass to the third line we have used the weak formulation (11), Green's formula to the term $\int_{D} \mathbf{H}_{h} \cdot \operatorname{curl} \overline{\epsilon_{A}}$ combined with the boundary conditions on $\epsilon_{A}$ on $\Gamma$, and finally extended the domain of the integral $\int_{D_{c}} \mathbf{J}_{e, h} \cdot \overline{\left(j \omega \epsilon_{A}+\nabla \epsilon_{\varphi}\right)}$ recalling that $\left.\mathbf{J}_{e, h}\right|_{D_{n c}}=0$. By construction of $\mathbf{H}_{h}$, see 21],

$$
\begin{array}{r}
\epsilon_{A, \varphi}^{2}=\frac{j}{\omega} \int_{D_{c}}\left(\sigma \mathbf{E}_{h}-\mathbf{J}_{e, h}\right) \cdot \overline{\left(j \omega \epsilon_{A}+\nabla \epsilon_{\varphi}\right)}+\int_{D}\left(\mathbf{H}_{h}-\mu^{-1} \mathbf{B}_{h}\right) \cdot \operatorname{curl} \overline{\epsilon_{A}} \\
+\int_{D}\left(\mathbf{J}_{s}-\Pi_{h} \mathbf{J}_{s}\right) \cdot \overline{\epsilon_{A}}+\int_{D}\left(\mathbf{J}_{e, h}-\Pi_{h} \mathbf{J}_{e, h}\right) \cdot \bar{\epsilon}_{A},
\end{array}
$$

where the term $\int_{D} \mathbf{J}_{e, h} \cdot \nabla \overline{\epsilon_{\varphi}}$ vanishes since we apply Green's formula, remarking that $\mathbf{J}_{e, h}$ is divergence-free and that $\epsilon_{\varphi}$ can be extended outside of $D_{c}$ in order to have $\epsilon_{\varphi}=0$ on $\Gamma$. Let us estimate each term of the right hand-side of the relation 25.

(I) The first two terms of the right hand-side of 25 lead to the error estimator terms. Indeed, applying the (continuous and discrete) Cauchy-Schwarz inequality we obtain directly:

$$
\begin{aligned}
& \frac{j}{\omega} \int_{D_{c}}\left(\sigma \mathbf{E}_{h}-\mathbf{J}_{e, h}\right) \cdot \overline{\left(j \omega \epsilon_{A}+\nabla \epsilon_{\varphi}\right)}+\int_{D}\left(\mathbf{H}_{h}-\mu^{-1} \mathbf{B}_{h}\right) \cdot \operatorname{curl} \overline{\epsilon_{A}} \\
& \leq \sum_{T \in \mathcal{T}_{h}, T \subset D_{c}}\left\|\left(\frac{j}{\omega \sigma}\right)^{1 / 2}\left(\mathbf{J}_{e, h}-\sigma \mathbf{E}_{h}\right)\right\|_{T}\left\|\left(\frac{j \sigma}{\omega}\right)^{1 / 2}\left(j \omega \epsilon_{A}+\nabla \epsilon_{\varphi}\right)\right\|_{T} \\
& +\sum_{T \in \mathcal{T}_{h}}\left\|\mu^{1 / 2}\left(\mathbf{H}_{h}-\mu^{-1} \mathbf{B}_{h}\right)\right\|_{T}\left\|\mu^{-1 / 2} \operatorname{curl} \epsilon_{A}\right\|_{T} \\
& \leq\left(\sum_{T \in \mathcal{T}_{h}, T \subset D_{c}} \eta_{\text {elec }, T}^{2}\right)^{1 / 2}\left\|\left(\frac{j \sigma}{\omega}\right)^{1 / 2}\left(j \omega \epsilon_{A}+\nabla \epsilon_{\varphi}\right)\right\|_{D_{c}} \\
& +\left(\sum_{T \in \mathcal{T}_{h}} \eta_{\mathrm{elec}, T}^{2}\right)^{1 / 2} \| \mu^{-1 / 2} \operatorname{curl} \epsilon_{A} \leq \eta \epsilon_{A, \varphi},
\end{aligned}
$$

where for the last inequality we have used the definition of the local estimators (16) first and the discrete Cauchy-Schwarz inequality with the definition of the global estimator 22 secondly.

(II) Now we prove that the last two terms of the right hand-side of 25) yield the oscillating term $r$. In the following $C>0$ denotes a generic constant which 
does not depend on the mesh size and the gauge broken Raviart-Thomas space in $D$ is denoted by

$$
\widetilde{\mathcal{R} \mathcal{T}_{0, h}}(D)=\left\{\mathbf{F}_{h} \in \mathcal{R} \mathcal{T}_{0}\left(\mathcal{T}_{h}\right): \operatorname{div} \mathbf{F}_{h}=0\right\}
$$

Moreover, we use the Helmholtz decomposition of Lemma 2.4.1 in [13] (taking the parameter $\beta=1$ ):

$$
H_{0}(\operatorname{curl}, D)=\nabla H_{0}^{1}(D) \stackrel{\perp}{\oplus} \widetilde{X}(D),
$$

so that

$$
\mathbf{A}-\mathbf{A}_{h}=\nabla \phi+\epsilon_{\perp}
$$

with $\phi \in H_{0}^{1}(\Omega)$ and $\epsilon_{\perp} \in \tilde{X}(D)$. From Theorem 3.5 of [18] there exists $\delta \in$ $(0,1]$ (depending on the geometry of $D$ ) and a constant $C>0$ such that $\epsilon_{\perp} \in$ $\left(H^{\delta}(D)\right)^{3}$ with the estimate

$$
\left\|\epsilon_{\perp}\right\|_{\delta, D} \leq C\left(\left\|\operatorname{curl} \epsilon_{\perp}\right\|+\left\|\operatorname{div} \epsilon_{\perp}\right\|\right) .
$$

Since $\epsilon_{\perp} \in \tilde{X}(D)$, the last term vanishes, so that:

$$
\left\|\epsilon_{\perp}\right\|_{\delta, D} \leq C\left\|\operatorname{curl} \epsilon_{\perp}\right\|
$$

Using the decomposition (27) for $\epsilon_{A}=\mathbf{A}-\mathbf{A}_{h}$, we get

$$
\int_{D}\left(\mathbf{J}_{s}-\Pi_{h} \mathbf{J}_{s}\right) \cdot \overline{\epsilon_{A}}=\underbrace{\int_{D}\left(\mathbf{J}_{s}-\Pi_{h} \mathbf{J}_{s}\right) \cdot \overline{\nabla \phi}}_{=0}+\int_{D}\left(\mathbf{J}_{s}-\Pi_{h} \mathbf{J}_{s}\right) \cdot \overline{\epsilon_{\perp}},
$$

where the first term in the right hand-side vanishes applying Green's formula and recalling that $\mathbf{J}_{s}-\Pi_{h} \mathbf{J}_{s}$ is divergence-free and that $\phi$ vanishes on $\Gamma$. Let us introduce $I_{\mathrm{RT} 0} \epsilon_{\perp} \in \widetilde{\mathcal{R} \mathcal{T}_{0, h}}(D)$ the $\mathcal{R} \mathcal{T}_{0}$-interpolant of $\epsilon_{\perp}$, thus we have

$$
\int_{D}\left(\mathbf{J}_{s}-\Pi_{h} \mathbf{J}_{s}\right) I_{\mathrm{RT} 0} \epsilon_{\perp}=0
$$

therefore 29$)$ becomes

$$
\int_{D}\left(\mathbf{J}_{s}-\Pi_{h} \mathbf{J}_{s}\right) \cdot \overline{\epsilon_{A}}=\int_{D}\left(\mathbf{J}_{s}-\Pi_{h} \mathbf{J}_{s}\right) \cdot \overline{\left(\epsilon_{\perp}-I_{\mathrm{RT} 0} \epsilon_{\perp}\right)} .
$$


Since $\epsilon_{\perp} \in\left(H^{\delta}(D)\right)^{3} \cap H(\operatorname{div}, D)$, Lemma 3.3 of [19] ensures that there exists a constant $C>0$ such that

$$
\left\|\epsilon_{\perp}-I_{\mathcal{R} \mathcal{T}_{0}} \epsilon_{\perp}\right\| \leq C\left(h^{\delta}\left\|\epsilon_{\perp}\right\|_{\delta}+h\left\|\operatorname{div} \epsilon_{\perp}\right\|\right)=C h^{\delta}\left\|\epsilon_{\perp}\right\|_{\delta} \leq C h^{\delta}\left\|\operatorname{curl} \epsilon_{\perp}\right\|,
$$

where we have used the divergence free property of $\epsilon_{\perp}$ to state the equality and 28 for the last inequality. Thanks to the Cauchy-Schwarz inequality and estimate (31), (30) is estimated as follows:

$$
\begin{array}{r}
\int_{D}\left(\mathbf{J}_{s}-\Pi_{h} \mathbf{J}_{s}\right) \cdot \overline{\epsilon_{A}} \leq C h^{\delta}\left\|\mathbf{J}_{s}-\Pi_{h} \mathbf{J}_{s}\right\|\left\|\operatorname{curl} \epsilon_{\perp}\right\| \\
\leq C \mu_{\max }^{1 / 2} \underbrace{h^{\delta}\left(\sum_{T \in \mathcal{T}_{h}}\left\|\mathbf{J}_{s}-\Pi_{h} \mathbf{J}_{s}\right\|_{T}^{2}\right)^{1 / 2}}_{\operatorname{osc}\left(\mathbf{J}_{s}\right)}\left\|\mu^{-1 / 2} \operatorname{curl} \epsilon_{A}\right\|,
\end{array}
$$

where for the last inequality we have used definition (27) to express $\epsilon_{\perp}$.

The same arguments used above for the source term yield:

$$
\begin{aligned}
& \int_{D}\left(\mathbf{J}_{e, h}-\Pi_{h} \mathbf{J}_{e, h}\right) \cdot \overline{\epsilon_{A}} \\
& \leq C \mu_{\max }^{1 / 2} \underbrace{h^{\delta}\left(\sum_{T \in \mathcal{T}_{h}}\left\|\mathbf{J}_{e, h}-\Pi_{h} \mathbf{J}_{e, h}\right\|_{T}^{2}\right)^{1 / 2}}_{\operatorname{osc}\left(\mathbf{J}_{e, h}\right)}\left\|\mu^{-1 / 2} \operatorname{curl} \epsilon_{A}\right\| .
\end{aligned}
$$

(III) Applying estimates (26), (32) and (33) to the identity (25), the upper bound 23 is proved.

(IV) Let us show that $r$ represents a higher order term. If we suppose $\mathbf{J}_{s} \in$ $\left(H^{1}(D)\right)^{3}$, then, by scaling arguments, $\operatorname{osc}\left(\mathbf{J}_{s}\right) \leq C h^{1+\delta}|| \mathbf{J}_{s} \|_{1, D}$, which means that it is a higher order term. Let us show that $\operatorname{osc}\left(\mathbf{J}_{e, h}\right)$ is also a higher order term. A scaling argument on each element $T$, gives $\left\|\mathbf{J}_{e, h}-I_{\mathrm{RT0}} \mathbf{J}_{e, h}\right\|_{T} \leq$ ${ }_{135} C h_{T}\left\|\nabla \mathbf{J}_{e, h}\right\|_{T}$, therefore, from the definition of the projection onto $\mathcal{R} \mathcal{T}_{0, h}$, we 
get

$$
\begin{aligned}
\left\|\mathbf{J}_{e, h}-\Pi_{h} \mathbf{J}_{e, h}\right\|^{2} & \leq \sum_{w_{h} \in \mathcal{R} \mathcal{T}_{0, h}(D)}^{\min } \sum_{T \in \mathcal{T}_{h}}\left\|\mathbf{J}_{e, h}-w_{h}\right\|_{T}^{2} \leq \sum_{T \in \mathcal{T}_{h}}\left\|\mathbf{J}_{e, h}-I_{\mathrm{RT0}} \mathbf{J}_{e, h}\right\|_{T}^{2} \\
& \leq C \sum_{T \in \mathcal{T}_{h}} h_{T}^{2}\left\|\nabla \mathbf{J}_{e, h}\right\|_{T}^{2} \\
& \leq C \sum_{T \in \mathcal{T}_{h}} h_{T}^{2}\left(\left\|\nabla\left(\mathbf{J}_{e, h}-\sigma \mathbf{E}_{h}\right)\right\|_{T}^{2}+\left\|\nabla \sigma \mathbf{E}_{h}\right\|_{T}^{2}\right)
\end{aligned}
$$

where at last we have used the triangle inequality. Let us estimate the first term of the right-hand side of (34): firstly, thanks to an inverse inequality [20] and, secondly, thanks to the local lower bound (37) (stated in Theorem 4.3), we have the estimate

$$
\begin{aligned}
& \left\|\nabla\left(\mathbf{J}_{e, h}-\sigma \mathbf{E}_{h}\right)\right\|_{T} \leq C h_{T}^{-1}\left\|\left(\mathbf{J}_{e, h}-\sigma \mathbf{E}_{h}\right)\right\|_{T} \\
& \leq C h_{T}^{-1} \omega^{1 / 2} \max _{T^{\prime} \in \omega_{T}} \sigma_{T^{\prime}}^{1 / 2} \sum_{T^{\prime} \in \omega_{F}: F \subset \partial T}\left\|\left(\frac{j \sigma}{\omega}\right)^{1 / 2}\left(j \omega \epsilon_{A}+\nabla e_{\varphi}\right)\right\|_{T^{\prime}}
\end{aligned}
$$

where

$$
\omega_{F}=\bigcup_{F \in \partial T} T \text { and } \omega_{T}=\bigcup_{T^{\prime} \subset \omega_{F}: F \subset T} T^{\prime}
$$

represent respectively the patch associated with the face $F$ and the patch associated with the element $T$. For the second term of the right-hand side of (34), we use Lemma 4.1 of [21] which ensures that:

$$
\left\|\nabla \sigma \mathbf{E}_{h}\right\|_{T}=\left\|\nabla \sigma\left(j \omega \mathbf{A}_{h}+\nabla \varphi_{h}\right)\right\|_{T} \leq \mu_{T}^{1 / 2}\left\|\mu^{-1 / 2} \operatorname{curl} \mathbf{A}_{h}\right\|_{T} .
$$

Finally, from the defintion of $\operatorname{osc}\left(\mathbf{J}_{e, h}\right)$, applying inequality (34). To pass to the second line and inequalities (35) and (36) to pass to the third line, we obtain:

$$
\begin{aligned}
\operatorname{osc}\left(\mathbf{J}_{e, h}\right)^{2} & \leq h^{2 \delta} \sum_{T \in \mathcal{T}_{h}}\left\|\Pi_{h} \mathbf{J}_{e, h}-\mathbf{J}_{e, h}\right\|_{T}^{2} \\
& \leq C h^{2 \delta}\left(\sum_{T \in \mathcal{T}_{h}} h_{T}^{2}\left\|\nabla\left(\mathbf{J}_{e, h}-\sigma\left(j \omega \mathbf{A}_{h}+\nabla \varphi_{h}\right)\right)\right\|_{T}^{2}+\left\|\nabla \sigma\left(j \omega \mathbf{A}_{h}+\nabla \varphi_{h}\right)\right\|_{T}^{2}\right) \\
& \leq C h^{2 \delta}\left(\left\|\left(\frac{j \sigma}{\omega}\right)^{1 / 2}\left(j \omega \epsilon_{A}+\nabla \epsilon_{\varphi}\right)\right\|_{D_{c}}^{2}+h^{2}\left\|\mu^{-1 / 2} \operatorname{curl} \mathbf{A}_{h}\right\|^{2}\right) \\
& \leq C h^{2 \delta}\left(\left\|\left(\frac{j \sigma}{\omega}\right)^{1 / 2}\left(j \omega \epsilon_{A}+\nabla \epsilon_{\varphi}\right)\right\|_{D_{c}}^{2}+h^{2}\left\|\mathbf{J}_{s}\right\|^{2}\right),
\end{aligned}
$$


where the last inequality follows directly from the weak formulation (11). Therefore $\operatorname{osc}\left(\mathbf{J}_{e, h}\right)$ is a higher order term.

Theorem 4.3. Let us suppose that $\mathbf{J}_{s} \in\left(L^{2}(D)\right)^{3}$ and that $\mathbf{J}_{e, h} \in \mathcal{R} \mathcal{T}_{1, h}$ satisfies (20) and is zero in $D_{n c}$. Then the following lower bounds hold:

$$
\begin{gathered}
\eta_{\text {elec }, T} \leq C \max _{T^{\prime} \in \omega_{T}}\left(\frac{\sigma_{T^{\prime}}}{\sigma_{T}}\right)^{1 / 2} \sum_{T^{\prime} \in \omega_{F}, F \subset \partial T}\left\|\left(\frac{j \sigma}{\omega}\right)^{1 / 2}\left(j \omega \epsilon_{A}+\nabla \epsilon_{\varphi}\right)\right\|_{T^{\prime}}, \\
\left(\sum_{T \in \mathcal{T}_{h}} \eta_{\operatorname{magn}, T}^{2}\right)^{1 / 2} \leq C\left(\sigma_{\max } \mu_{\max }\right)^{1 / 2}\left\|\left(\frac{j \sigma}{\omega}\right)^{1 / 2}\left(j \omega \epsilon_{A}+\nabla \epsilon_{\varphi}\right)\right\|_{D_{c}} \\
+\left\|\mu^{-1 / 2} \operatorname{curl} \epsilon_{A}\right\|+\mu_{\max }^{1 / 2}\left(\operatorname{osc}\left(\mathbf{J}_{s}\right)+\operatorname{osc}\left(\mathbf{J}_{e, h}\right)\right),
\end{gathered}
$$

where $C>0$ represents a constant which does not depend on the mesh size (but on the regularity of the mesh).

The proof is an application of standard lower bound techniques for a posteriori error estimators 20] (or [13] for the electromagnetism framework). We remark that for the electric error estimator the lower bound is local, see (37), that is a suitable property for local mesh adaptation. For the magnetic error estimator the lower bound is global, see (38), this is due to the use of a global estimation linked to Lemma 3.2 and the second Strang Lemma. For more details see Theorem 2.4.5 of [22. A way to overcome this drawback could be to build the admissible magnetic field $\mathbf{H}_{h}$ solving local problems on dual meshes, e.g. in the same spirit of [23].

As a direct consequence of Theorems 4.2 and 4.3 , we state:

Corollary 4.4. Let us suppose that $\mathbf{J}_{s} \in\left(H^{1}(D)\right)^{3}$ and that $\mathbf{J}_{e, h} \in \mathcal{R} \mathcal{T}_{1, h}$ satisfies 20 and is zero in $D_{n c}$. Then there exists a contant $C>0$ which does not depend on the mesh size such that

$$
C \eta_{\text {flux }} \leq \epsilon_{A, \varphi} \leq \eta_{\text {flux }} \text { up to some higher order terms. }
$$

\section{Numerical tests}

This section starts with some practical remarks about the computation of the error estimator $\eta_{\text {flux }}$. Afterwords, we present an analytical benchmark test 
in order to validate the theorical results. The section ends with a physical benchmark test to show the efficiency of the equilibrated error estimators. Another physical benchmark test can be found in 24 .

\subsection{Analytical benchmark}

In this paragraph the two estimators are validated using the same benchmark test proposed in 11. The geometrical domain is showed in Fig. 1 . $D=$ $[-2.5,5] \times[-2,2] \times[-2,2], D_{c}=[2,4] \times[-1,1] \times[-1,1]$ and $D_{s}=[-1,1]^{3}$. A density current $\mathbf{J}_{s}$ is imposed in $D_{s}$ such that the exact solution $(\mathbf{A}, \varphi)$ is

\footnotetext{
${ }^{2}$ http://code-carmel.univ-lille1.fr
} 
known, indeed we chose

$$
\mathbf{A}=\operatorname{curl}\left(\begin{array}{c}
f(x, y, z) \\
0 \\
0
\end{array}\right) \quad \text { in } D,
$$

where

$$
f(x, y, z)=\left\{\begin{array}{lr}
\left(x^{2}-1\right)^{4}\left(y^{2}-1\right)^{4}\left(z^{2}-1\right)^{4} & \text { in } D_{s} \\
0 & \text { otherwise }
\end{array}\right.
$$

and $\varphi \equiv 0$ in $D_{c}$. Thus the estimators $\eta_{\text {flux }}$ and $\eta_{\text {dual }}$ and also the errors which they estimate are computable: respectively $\epsilon_{A, \varphi}$ and $\left(\epsilon_{A, \varphi}^{2}+\epsilon_{T, \Omega}^{2}\right)^{1 / 2}$. The conductivity and the permeability are fixed to one and the frequence is fixed to $f=50 \mathrm{~Hz}$. Choosing four meshes uniformly refined, Fig. 2 displays the convergence in the log-log scale of the estimators and the estimated errors with respect to the Degrees of Freedom (DoF). The first remark is that the estimated errors $\epsilon_{A, \varphi}$ and $\left(\epsilon_{A, \varphi}^{2}+\epsilon_{T, \Omega}^{2}\right)^{1 / 2}$ have the expected rate of convergence for a regular finite element solution, that is $-1 / 3$. Moreover, both estimators have the same rate of convergence and the same order of magnitude as the corresponding estimated errors.

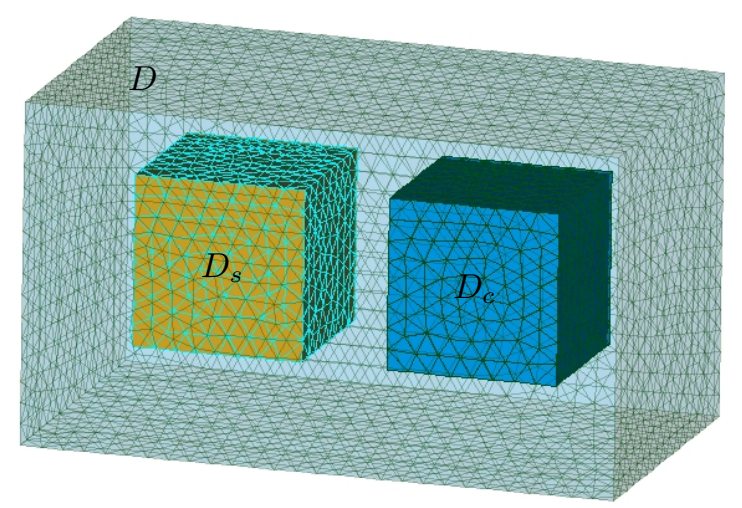

Figure 1: Geometrical model and one of the regular meshes used for the analytical test. 


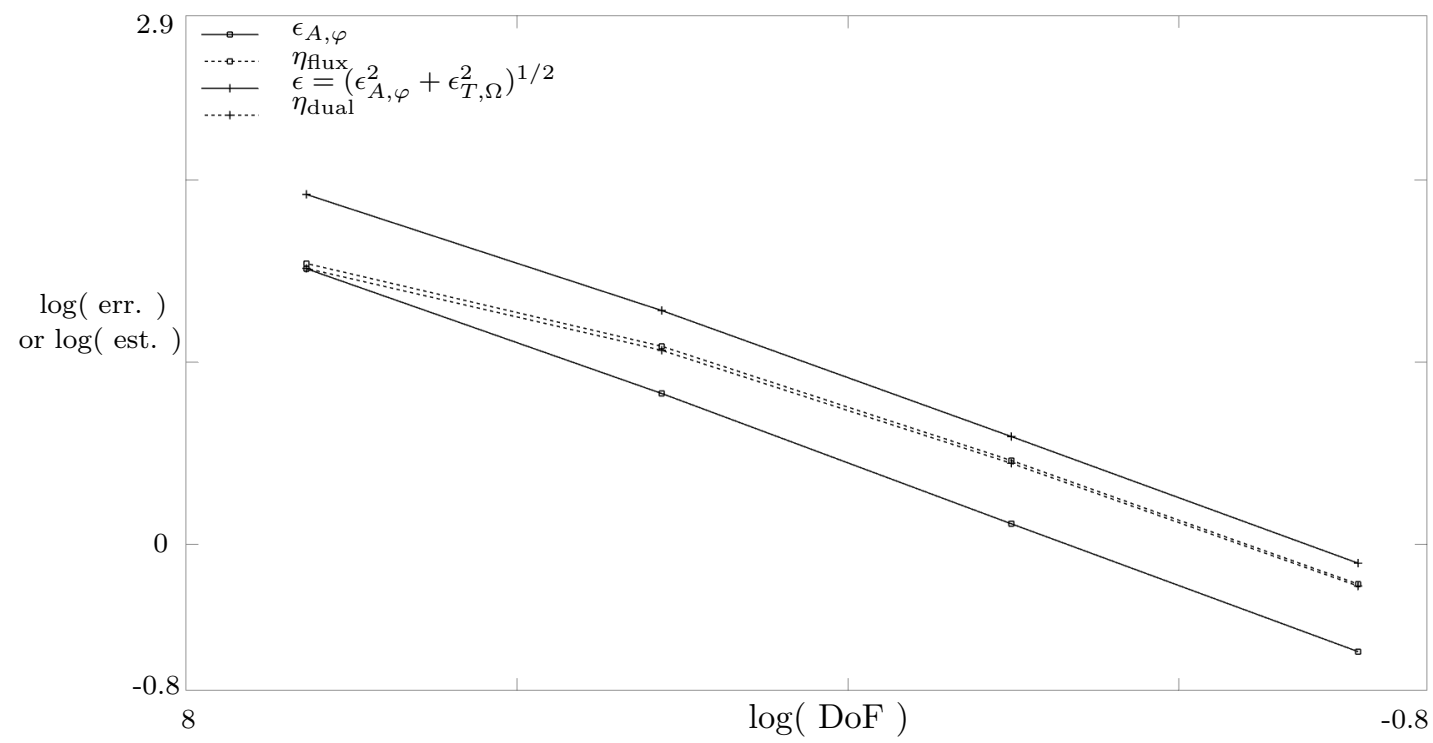

Figure 2: Rate of convergence in the log-log scale of the estimators and their errors estimated with respect to the DoF $=6172,52829,437081,3555697$. 


\subsection{Physical benchmark}

In order to compare qualitatively the two estimators, the physical benchmark case Team Workshop 7 is considered 3 The structure is composed of an

195 The conductor plates has a conductivity $\sigma$ equal to $3.526 \times 10^{7} \mathrm{~S} / \mathrm{m}$ and in the whole domain the permeability is fixed to $\mu=4 \pi \times 10^{-7} \mathrm{H} / \mathrm{m}$. The coil is fed by a sinusoidal voltage at the frequency of $50 \mathrm{~Hz}$, so that eddy current is created in the plate. The eddy current is distributed geometrically and is more important near the singularity of the boundary, as expected from the physical point of view, see Fig. 3b.

We consider four tetrahedral meshes uniformly refined, with respectively 12183, 25843, 50438, 598480 mesh elements. Fig. 4 represents the Ohmic losses and the magnetic energy of the $\mathbf{A}-\varphi$ and $\mathbf{T}-\Omega$ formulations computed with respect to the four meshes. In both cases, as expected, refining the mesh, the two solutions converge towards the same solution.

Fig. 5 depicts the rate of convergence of the two estimators in the log-log scale with respect to the DoF. The convergence is guaranteed and we notice that, having a singular benchmark test, the rate of convergence is a little bit less than the one expected for the regular benchmark case.

Fig. 6a represents the distributions in the plate and in the coil of the estimator $\eta_{\text {flux }}$ and Fig. 6b of the estimator $\eta_{\text {dual }}$. Both estimators detect a higher error in regions where eddy current are located. Even if we do not dispose of a local lower bound for the error $\epsilon_{A, \varphi}$ by the estimator $\eta_{\text {flux }}$, from these figures 215 we can see a good agreement between the two estimators on each tetrahedron. In other terms, we observe a numerical local efficiency of $\eta_{\text {flux }}$.

\footnotetext{
${ }^{3}$ http://www.compumag.org/jsite/images/stories/TEAM/problem7.pdf
} 

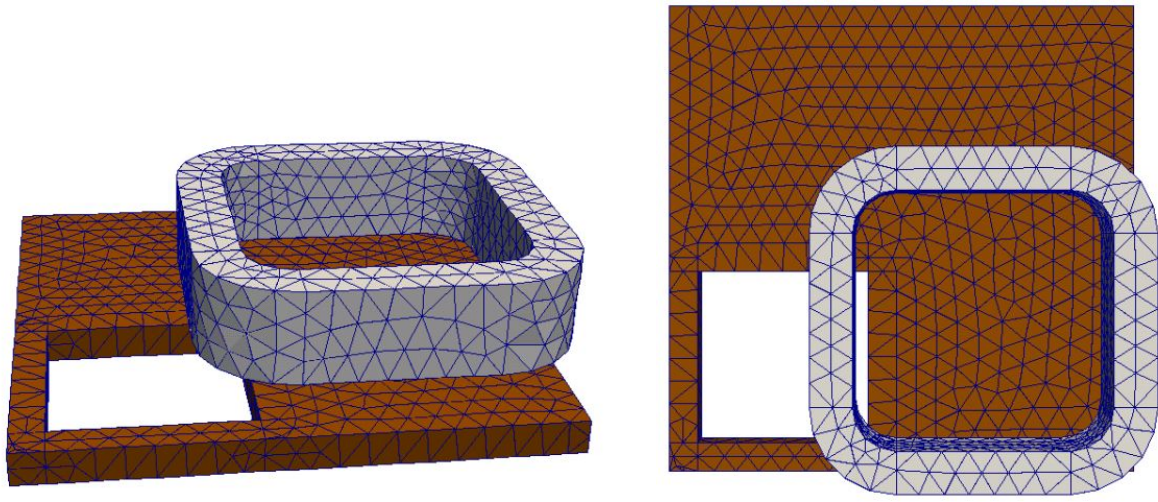

(a) Mesh with 50438 elements

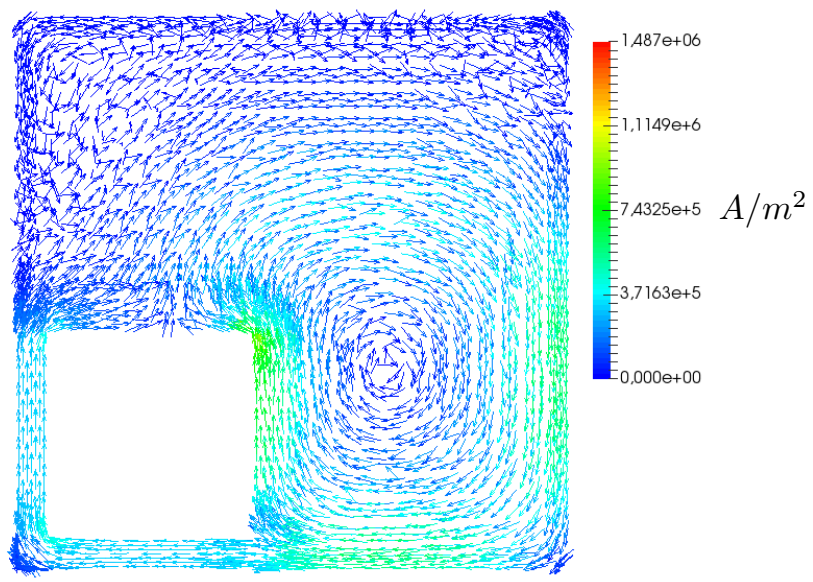

(b) Eddy currents in the plate

Figure 3: Example of a uniform mesh (figure a)) for the benchmark case Team Workshop 7. The structure is composed of an asymmetrical conductor with a hole and a coil. The density current imposed in the coil (at a frequency of $50 \mathrm{~Hz}$ ) produces eddy currents in the plate (figure b)). 

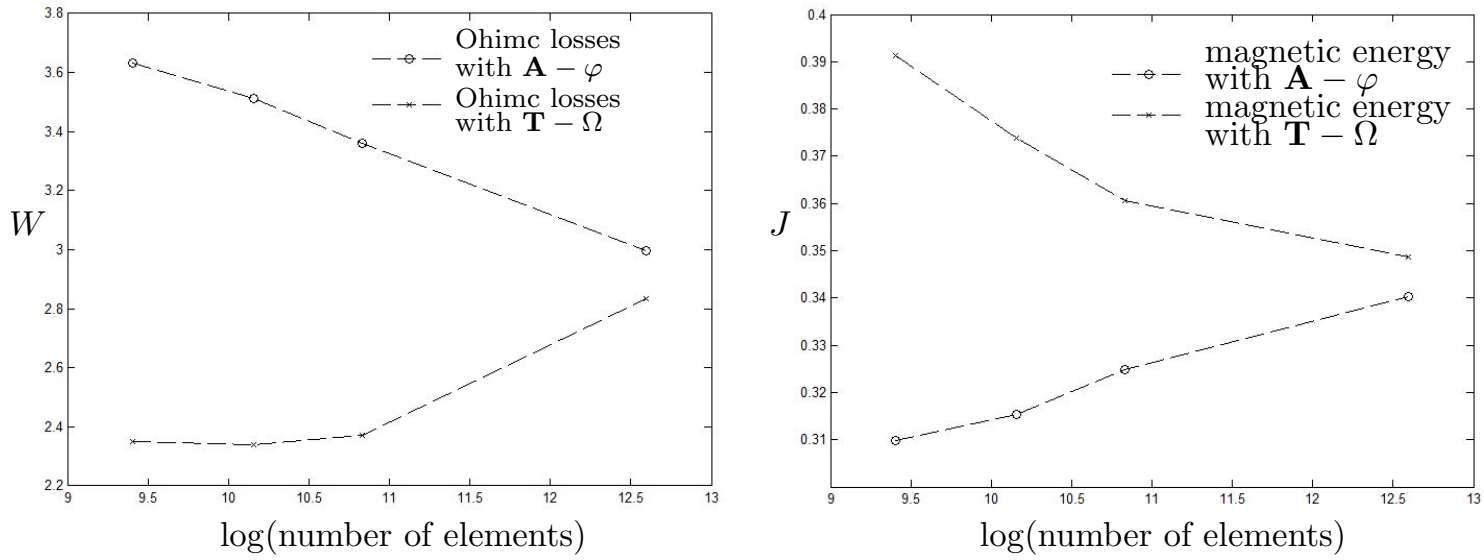

Figure 4: Ohmic losses (Watt) and magnetic energy (Joule) computed for the two formulations, $\mathbf{A}-\varphi$ and $\mathbf{T}-\Omega$, with respect to the number of mesh elements.

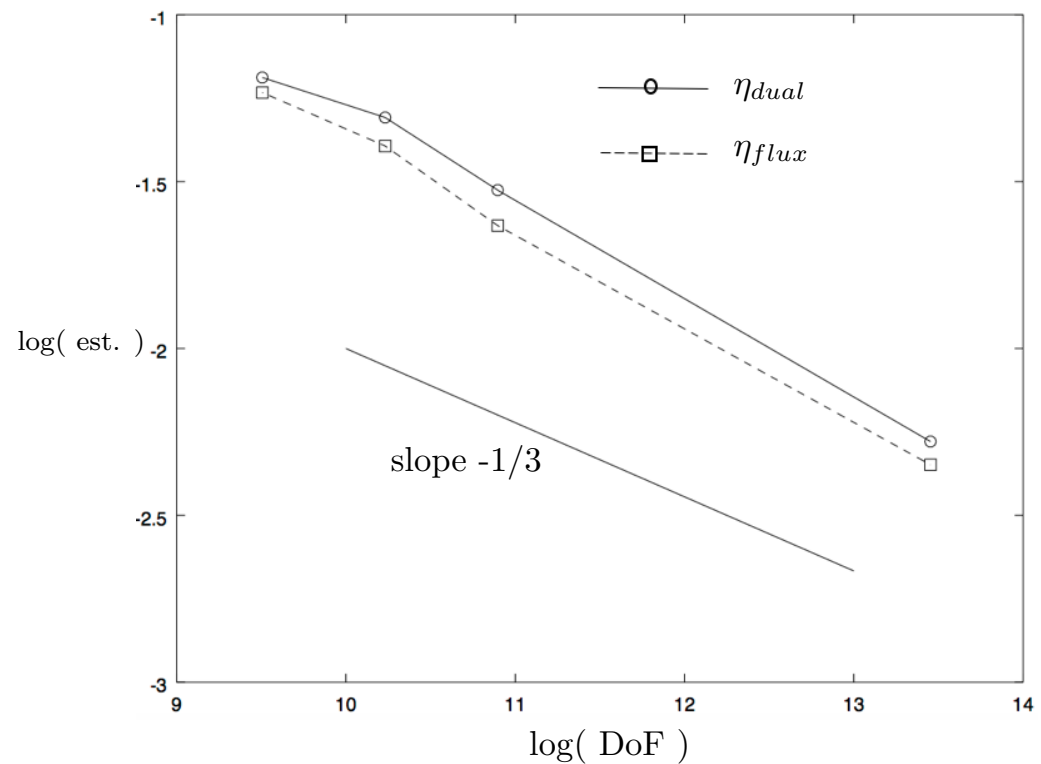

Figure 5: Log-log plot of the convergence of the two error estimators $\eta_{\text {dual }}$ and $\eta_{\text {flux }}$ with respect to the DoF for four different meshes. 

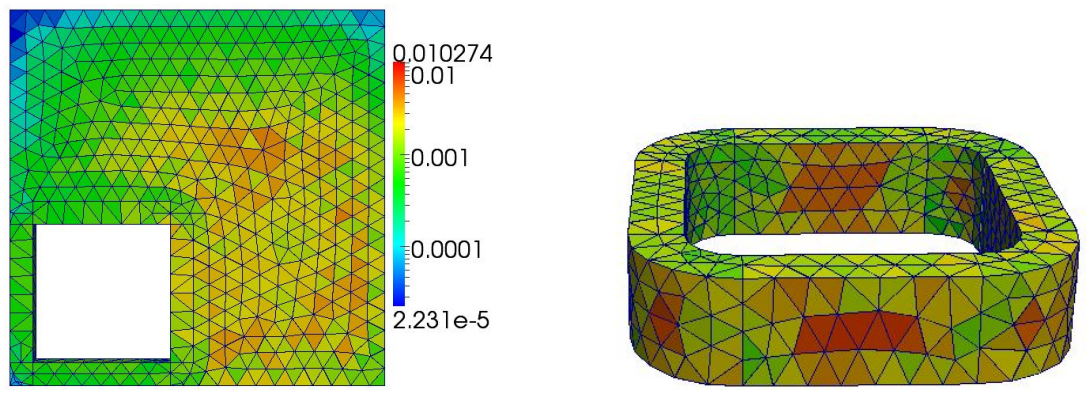

(a) Distribution of $\eta_{f l u x}$
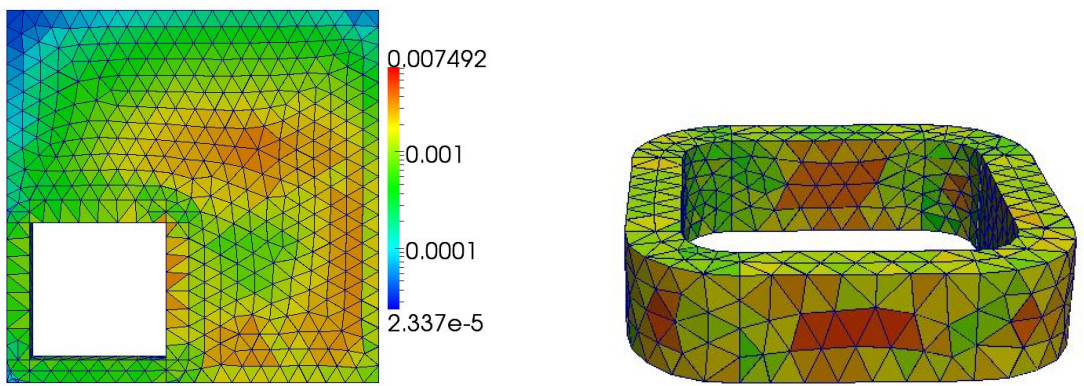

(b) Distribution of $\eta_{\text {dual }}$

Figure 6: Map of the two error estimators in the plate and in the coil for the computation with 50438 mesh elements. 


\section{Conclusions}

We have presented two guaranteed a posteriori error estimators for the eddy current problems and proved the global upper bound for the error by the estimator based on the flux reconstruction technique. The numerical results validate the theoretical predictions and show that both estimators could be used to drive a mesh refinement. Moreover, globally they quantify accurately the error, thus they could be employed as stopping criterion in an adaptive mesh refinement algorithm. A natural extension of this work consists in developing an equilibrated error estimator for the $\mathbf{T}-\Omega$ formulation uniquely.

\section{Acknowledgments}

This work was supported by EDF R\&D, and it is realized under the MEDEE project with financial Assistance of the European Regional Development Fund and the region Nord-Pas-de-Calais. We acknowledge Antoine Pierquin for his fruitful contribution for the numerical simulations.

\section{Bibliography}

[1] P. Monk, A posteriori error indicators for Maxwell's equations, J. Comput. Appl. Math. 100 (2) (1998) 173-190. doi:10.1016/S0377-0427(98) 00187-3.

URL http://dx.doi.org/10.1016/S0377-0427(98)00187-3

[2] E. Creusé, S. Nicaise, Z. Tang, Y. Le Menach, N. Nemitz, F. Piriou, Residual-based a posteriori estimators for the $\mathbf{A}-\varphi$ magnetodynamic harmonic formulation of the Maxwell system, Math. Models Methods Appl. Sci. 22 (5) (2012) 1150028, 30. doi:10.1142/S021820251150028X.

URL http://dx.doi.org/10.1142/S021820251150028X 
[3] E. Creusé, S. Nicaise, Z. Tang, Y. Le Menach, N. Nemitz, F. Piriou, Residual-based a posteriori estimators for the $\mathbf{T} / \Omega$ magnetodynamic harmonic formulation of the Maxwell system, Int. J. Numer. Anal. Model. 10 (2) (2013) 411-429.

q [4] R. Beck, R. Hiptmair, R. H. W. Hoppe, B. Wohlmuth, Residual based a posteriori error estimators for eddy current computation, M2AN Math. Model. Numer. Anal. 34 (1) (2000) 159-182. doi:10.1051/m2an:2000136 URL http://dx .doi .org/10.1051/m2an:2000136

[5] J. Rikabi, C. Bryant, E. Freeman, Error-based derivation of complementary formulations for the eddy-current problem, Physical Science, Measurement and Instrumentation, Management and Education - Reviews, IEE Proceedings A 135 (4) (1988) 208-216.

[6] C. Li, L. Ren, A. Razek, An approach to adaptive mesh refinement for three-dimensional eddy-current computations, IEEE Transactions on Magnetics 30 (1) (1994) 113-117. doi:10.1109/20.272523.

[7] N. Golias, T. Tsiboukis, A. Bossavit, Constitutive inconsistency: rigorous solution of Maxwell equations based on a dual approach, Magnetics, IEEE Transactions on 30 (5) (1994) 3586-3589. doi:10.1109/20.312714.

[8] J. F. Remacle, P. Dular, A. Genon, W. Legros, A posteriori error estimation and adaptive meshing using error in constitutive relation, IEEE Transactions on Magnetics 32 (3) (1996) 1369 - 1372.

[9] J.-F. Remacle, C. Geuzaine, P. Dular, H. Hedia, W. Legros, Error estimation based on a new principle of projection and reconstruction, IEEE Transactions on Magnetics 34 (5) (1998) 3264 - 3267.

[10] F. Marmin, S. Clénet, F. Bouillault, F. Piriou, Calculation of complementary solutions in 2d finite element method application to error estimation, IEEE Transactions on Magnetics 36 (4) (2000) 1583 - 1587. 
[11] E. Creusé, S. Nicaise, R. Tittarelli, A guaranteed equilibrated error es-

[12] M. Ainsworth, J. T. Oden, A posteriori error estimation in finite element analysis, Pure and Applied Mathematics (New York), Wiley-Interscience [John Wiley \& Sons], New York, 2000. doi:10.1002/9781118032824. URL http://dx.doi.org/10.1002/9781118032824

[13] S. Cochez-Dhondt, Méthodes d'éléments finis et estimations d'erreur a posteriori, Ph.D. thesis, Université de Valenciennes et du Hainaut-Cambrésis (2007).

[14] D. Braess, J. Schöberl, Equilibrated residual error estimator for edge n elements, Math. Comp. 77 (262) (2008) 651-672. doi:10.1090/ S0025-5718-07-02080-7.

URL http://dx.doi.org/10.1090/S0025-5718-07-02080-7

[15] Z. Tang, Y. Le Menach, E. Creuse, S. Nicaise, F. Piriou, N. Nemitz, Residual and equilibrated error estimators for magnetostatic problems solved by finite element method, IEEE Transactions on Magnetics 49 (5).

[16] S. Cochez-Dhondt, S. Nicaise, A posteriori error estimators based on equilibrated fluxes, Comput. Methods Appl. Math. 10 (1) (2010) 49-68. doi:10.2478/cmam-2010-0002 URL http://dx .doi .org/10.2478/cmam-2010-0002

[17] D. Boffi, F. Brezzi, M. Fortin, Mixed finite element methods and applications, Vol. 44 of Springer Series in Computational Mathematics, Springer, Heidelberg, 2013. doi:10.1007/978-3-642-36519-5. URL http://dx.doi .org/10.1007/978-3-642-36519-5

[18] M. Costabel, M. Dauge, S. Nicaise, Singularities of Maxwell interface problems, M2AN Math. Model. Numer. Anal. 33 (3) (1999) 627-649. 
[19] I. G. Graham, R. Scheichl, E. Ullmann, Mixed finite element analysis of lognormal diffusion and multilevel Monte Carlo methods, Stoch.

n Partial Differ. Equ. Anal. Comput. 4 (1) (2016) 41-75. doi:10.1007/ s40072-015-0051-0.

URL http://dx.doi .org/10.1007/s40072-015-0051-0

[20] R. Verfürth, A review of a posteriori error estimation and adaptive meshrefinement techniques, Chichester and Stuttgart : Wiley and Teubner, Amsterdam, 1996.

305 [21] S. Nicaise, Edge elements on anisotropic meshes and approximation of the

口 Maxwell equations, SIAM J. Numer. Anal. 39 (3) (2001) 784-816. doi: $10.1137 / \mathrm{S} 003614290036988 \mathrm{X}$

URL http://dx.doi.org/10.1137/S003614290036988X

[22] R. Tittarelli, Estimateurs d'erreur a posteriori pour les équations de Maxwell en formulation potentielle et temporelle., Ph.D. thesis, Université de Lille 1 Sciences et Technologies (2016).

[23] M. Vohralík, Guaranteed and fully robust a posteriori error estimates 1. for conforming discretizations of diffusion problems with discontinuous

1. coefficients, J. Sci. Comput. 46 (3) (2011) 397-438. doi:10.1007/ 315 s10915-010-9410-1.

URL http://dx.doi.org/10.1007/s10915-010-9410-1

[24] R. Tittarelli, Y. Le Menach, F. Piriou, E. Creusé, S. Nicaise, J. Ducreux, Comparison of numerical error estimators for eddy current problems solved by fem, IEEE Transactions on Magnetics, 2017, in press.

320 [25] Y. Le Menach, S. Clénet, F. Piriou, Numerical model to discretise source fields in 3d finite element method, IEEE Transactions on Magnetics 36 (2000) 676-679.

[26] A. Pierquin, Y. Le Menach, J.-Y. Roger, L. Chevallier, Imposition d'un courant uniforme dans un conducteur, Numelec, Marseille, 3-5 juillet 2012. 
[27] Z. Badics, Z. J. Cendes, Source field modeling by mesh incidence matrices, IEEE Transactions on Magnetics 43 (4) (2007) 1241-1244. doi:10.1109/ TMAG.2006.890967. 